\title{
Passive immunotherapy for ebola virus infection
}

Volume I Issue 4 - 2014

\section{Opinion}

The recent outbreak of infections with Ebola virus (EV) in West Africa caused already over 4000 deaths and is rapidly increasing. In the interim while there are no effective drugs against this lethal virus it would be reasonable to explore the wider use of passive immunotherapy (PIT) based on the reported study of Mupapa et al. ${ }^{1}$ during the 1995 Ebola outbreak in the Democratic Republic of Congo. During this epidemic blood was collected from 5 convalescent patients who had anti-EV antibodies and then used to transfuse 8 seriously ill patients who ranged in age 12-54. The treated patients had severe asthenia, four had also hemorrhagic manifestation and two became comatose. Each individual received only a single infusion: seven of the 8 recovered. The overall fatality rate at the same hospital was $80 \%$ (249/311). In the absence of any widely available alternative to reduce the high mortality rate, treatment based on Mupapa's observation deserves to be exploited.

Plasma from people who recovered from the infection can be readily collected: and such anti-EV serum could be used to treat not only the advanced patients but also virus-infected pre symptomatic individuals during the incubation period. Hyper immune serum can lead to the recovery of terminal patients, if given earlier it might prevent the development of the disease by reducing the viral load and assisting the body's immune system to cope with the infection. Furthermore, if the observation of Mupapa et al. ${ }^{2}$ that a single infusion is correct, there may be a place for therapeutic immune anti sera prepared in large animals, like the anti-diphtheria and anti-tetanus used during the early part of last century. Early studies with equine immunoglobulin have already shown some activity in suppressing EV-viremia and delaying disease onset and death in non human primates. ${ }^{2}$

While the development of human monoclonal blocking antibodies to EV would be the ideal treatment until effective antiviral drugs are readily available for the thousands infected, PIT is likely to be the only beneficial treatment that can be offered without delay. Mupapa et al. ${ }^{2}$ study was published in 1999 and therefore it will not be unreasonable to assume that the 7 patients who recovered following a single PIT remained well and EV free. In 1985 we initiated PIT for AIDS patients in Cambridge using plasma from healthy HIV infected individuals which contain high levels of ant-HIV antibodies. ${ }^{3}$ Although we were able to demonstrate that extracellular HIV was neutralized and that there was a clinical benefit, when the monthly transfusions were stopped the patients deteriorated and died. ${ }^{4,5}$ The reason being that $\mathrm{HIV}$ is a retrovirus, namely its proviral DNA maintain a lifelong intracellular infection. In contrast $\mathrm{EV}$ infected individuals who recover

\author{
Abraham Karpas \\ Cambridge University emeritus, UK
}

Correspondence: Abraham Karpas, Cambridge University emeritus, 19 Wilberforce road Cambridge CB3 OEQ, UK, Tel 44-7766600835, Email ak1000I@cam.ac.uk

Received: November 07, 2014 | Published: November I8, 2014

appear to remain virus free. Since EV is not an airborne infection and those who recover are virus free, it should be possible to limit the spread of the virus. The addition of interferon might further improve the prognosis of infected individuals.

\section{Acknowledgments}

None.

\section{Conflicts of interest}

None.

\section{References}

1. Mupapa K, Massamba M, Kibadi K, et al. Treatment of Ebola Hemorrhagic Fever with Blood Transfusions from Convalescent patients. J Infect Dis. 1999;179Supp11:S18-S23.

2. Breman JG, Van der Groen G, Peters CJ, et al. International colloquium on Ebola virus research: Summary report. $J$ Infect Dis. 1997;176:1058-1063.

3. Karpas A, Gillson W, Bevan PC, et al. Lytic Infection by British AIDS virus and the development of rapid cell test for antiviral antibodies. Lancet. 1985;2(8457):695-697.

4. Abraham Karpas, Fergal Hill, Michael Youlet, et al. Effects of passive immunotherapy in patients with the acquired immunodeficiency syndrome-related complex and acquired immunodeficiency. Proc Natl Acad Sci. 1988;88: 9234-9237.

5. Karpas A, Human retroviruses in leukaemia and AIDS: reflections on their discovery, biology and epidemiology. Biol Rev Camb Philos Soc. 79(4):911-933. 